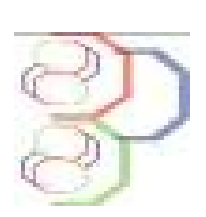

Journal of Applied Biosciences 116: 11532-11545

ISSN 1997-5902

\title{
Caractérisation agro-climatique du périmètre sucrier de Ferké 2 au Nord de la Côte d'Ivoire
}

\author{
Estelle A. KONAN¹, Crépin B. PÉNÉ1, Emmanuel DICK² \\ Sucaf-Cl, Direction R\&D, 01 BP1967 Abidjan 01, kadestkonan@yahoo.fr, bpene@sucafci.somdiaa.com, \\ UFR Bio-Sciences, Université FHB d'Abidjan-Cocody, 01 BPV34 Abidjan 01
}

Original submitted in on $15^{\text {th }}$ May 2017. Published online at www.m.elewa.org on $31^{\text {st }}$ August 2017 http://dx.doi.org/10.4314/jab.v116i1.2

\section{RÉSUMÉ}

Objectif : Le présent travail est une contribution aux efforts de recherche appliquée visant à accroître la productivité agricole dans les périmètres sucriers de Ferké par la caractérisation des apports d'eau de pluies au sein de Ferké 2 afin de pouvoir proposer des voies d'amélioration dans la conduite pratique des arrosages. Méthodologie et résultats : Les deux périmètres sucriers de Ferké sont situés au Nord de la Côte d'Ivoire Le climat de la région de Ferké est de type tropical sec avec deux saisons : l'une sèche, de novembre à avril et l'autre humide, de mai à octobre. La caractérisation de la pluviométrie a d'abord consisté à constituer une base de données comportant les 36 cumuls de pluies décadaires correspondant à chacune des 15 années considérées (1999 à 2014). Ensuite, à partir de cette base de données, il s'agissait de déterminer la répartition annuelle des pluies décadaires pour les années sèche, médiane et humide, à l'aide d'une analyse fréquentielle des pluies journalières enregistrées. La variabilité interannuelle de la pluviométrie a été caractérisée par l'indice de Nicholson, une variable centrée-réduite permettant d'atténuer les différences interannuelles afin de faire ressortir l'évolution de la pluviométrie et d'en dégager la tendance climatique pour une série pluviométrique donnée

Conclusion et application des résultats: II est ressorti de cette étude une répartition décadaire de la pluviométrie annuelle très différente selon que l'année est sèche, médiane ou humide avec les fréquences respectivement égales à 8 ans/10,5ans/10 et 2 ans/10. En outre, les variations de l'indice de Nicholson et de la moyenne mobile des pluies ont montré une atténuation de la pluviométrie sur la période allant de 1999 à 2014 . Sur la base de l'évapotranspiration potentielle calculée, le déficit pluviométrique cumulé sur la période sèche concernée par la pratique de l'irrigation était d'environ $200 \mathrm{~mm}$; celle-ci débute de novembre à mars.

Mots clés : climat tropical, analyse fréquentielle, bilan hydrique, évapotranspiration potentielle, front intertropical.

\section{ABSTRACT}

Agro-climatic characterization of Ferké 2 sugarcane plantations in northern Ivory Coast.

Objective: The present work is a contribution to applied research efforts aiming at increasing crop productivity on Ferké sugarcane plantations through characterization of Ferké 2 rainfall patterns, in order to recommend sound irrigation scheduling practices. Ferké climate is of tropical type with 2 seasons. One of them is dry which occurs from November to April, the other being wet, from May to October. Rainfall characterization required a database of 36 ten-day rainfall depths regarding each of all 15 years considered (1999-2014). From that 

la Côte d'Ivoire

database, rainfall frequency analysis was made to determine the annual ten-day rainfall distribution over the dry, wet as well as median years. Rainfall variability was estimated by the Nicholson index which is a centered variable that mitigates annual rainfall differences as to show the rainfall pattern and the climate trend.

Conclusion and application of results: It came out that rainfall distributions were very different depending on the year being dry, wet or median with the following frequencies: $8 \mathrm{yrs} / 10,2 \mathrm{yrs} / 10$ and $5 \mathrm{yrs} / 10$ respectively. Moreover, the Nicholson index variations and the running average rainfall showed lower rainfall patterns over the 1999-2010 period. Depending on the potential evapotranspiration calculated, the cumulated rainfall deficit over the dry season being concerned by the practice of irrigation, from November to June, was about 350-400 $\mathrm{mm}$ as compared to $650-700 \mathrm{~mm}$ estimated using pan evaporation data.

Key words: tropical climate, frequency analysis, water balance, evapotranspiration, intertropical front.

\section{INTRODUCTION}

La privatisation du secteur sucrier ivoirien en juillet 1997 visait à mettre fin, à moyen terme, à son manque récurrent de compétitivité lié aux coûts de production élevés du sucre de canne depuis sa création au début des années 1970. (Péné et al, 1997 ; Péné et Assa, 2003). Mais l'effort d'investissements structurants mis en œuvre par les nouveaux opérateurs sucriers dans le cadre de cette privatisation a dû connaître un fléchissement après le déclenchement de la crise socio-politique en septembre 2002 soit 5 années plus tard. II s'en est suivi, durant près d'une décennie, la mévente du sucre produit localement en raison des importations frauduleuses par les frontières nord du pays qui échappaient au contrôle des pouvoirs publics (Péné et al, 2013, 2016). Pour atténuer les conséquences économiques résultant de la réforme du régime sucrier entre les pays ACP et l'UE intervenue en 2006, celle-ci a mis en œuvre dans ces pays une politique de relance de la filière canne à sucre à travers des appuis financiers sectoriels (Kouamé et al, 2010 ; Péné et al, 2012a). En ce qui concerne la Côte d'Ivoire dont le secteur sucrier était si durement éprouvé, cet appui a été axé sur la redynamisation de la recherche agronomique appliquée pour accroître durablement la productivité agricole de 25 $\%$, réduire les coûts de production de $18 \%$ et permettre ainsi d'assurer la compétitivité du secteur (SOPEX-MSIRI, 2011). La sélection de variétés performantes tant pour la culture industrielle irriguée que pour la canne villageoise conduite en conditions pluviales, d'une part, et l'amélioration des pratiques culturales pour réduire les coûts d'exploitation, d'autre part, ont été définies comme axes prioritaires de recherche. Jusqu'à une date récente (campagne
2006/07), le matériel végétal cultivé dans les périmètres de la SucafCl était constitué de variétés obsolètes parmi lesquelles deux principales (Co 957 et NCo 376) avérées peu performantes en sucre (7-8 t/ha) occupaient 55 à $60 \%$ des superficies, soit 6000 à 6500 ha (Péné et Tuo, 2007 ; Péné et al, 2010). Cela expliquait en partie le plafonnement de la production sucrière à 92000 t/an avec parfois une baisse importante en année relativement sèche comme durant la campagne agricole 2007/08 avec 85500 t (Péné et Tuo, 2007). La tendance de la production sucrière à la hausse amorcée depuis la campagne sucrière 2008/09 en passant de $90300 \mathrm{t}$ jusqu'à près de $100000 \mathrm{t}$ en 2010/11, semble être en grande partie liée au développement de variétés plus productives en sucre que sont SP71-1406, SP70-1006, SP70-1143, SP71-8210, SP70-1423 et R579. Ce changement de profil variétal en cours s'est opéré progressivement, d'année en année, au détriment des principaux clones d'alors (Co957 et $\mathrm{NC} 0376$ ) et de bien d'autres plus faiblement représentées telles que Q75, Co440, FR8069, M31/45, R570 et R70/367 (Péné et al, 2012b, Zadi et al, 2017). La sélection variétale apparaît donc comme une composante essentielle dans l'accroissement de la productivité agricole au même titre que l'irrigation dans le contexte des périmètres sucrier de Ferké (Péné et Déa, 2000 ; Péné et al, 2001). C'est pourquoi dès la campagne sucrière 2006/07, une politique variétale dynamique, basée sur un schéma de sélection allégé à cycle court soit 6 ans au lieu de 11 voire 15 ans comme antérieurement et l'importation exclusive de variétés commerciales ou élites, a été mise en œuvre pour soutenir 2 plans triennaux d'investissements (2005- 
2007 et 2008-2010) pour la montée en production. Le double défi à relever porte sur la sélection de variétés de canne à sucre performantes qui soient résistantes, tolérantes ou moyennement tolérantes à la sécheresse aussi bien pour la culture pluviale (industrielle ou villageoise) que pour la culture industrielle irriguée où environ $40 \%$ des surfaces cultivées (soit 4000 ha) sont équipées de systèmes d'arrosage peu efficients (enrouleurs, couverture intégrale en quadrillage $18 \mathrm{~m} \times 24 \mathrm{~m}$, couverture partielle, goutte à goutte mal maintenu). L'étude vise à améliorer la productivité agricole par la caractérisation des apports d'eau de pluies et d'irrigations enregistrés au sein du périmètre sucrier de Ferké 2 afin de pouvoir proposer des voies d'amélioration dans la conduite pratique des

\section{MATÉRIEL ET MÉTHODES}

Situation géographique : Les deux périmètres sucriers de Ferké sont situés au Nord de la Côte d'Ivoire entre, d'une part, $9^{\circ} 20^{\prime}$ et $9^{\circ} 60^{\prime}$ de latitude nord et, d'autre part, $5^{\circ} 22^{\prime}$ et $5^{\circ} 40$ de longitude ouest, avec une altitude moyenne $325 \mathrm{~m}$ au-dessus du niveau de la mer. Ils sont limités à l'Ouest par le Bandama, à l'Est par la route nationale Tafiré - Ferké, au Sud par la rivière Farakwo et au Nord par la route nationale Ferké-Sinématiali (Fig 1). Climat prévalant dans la région de Ferké-Tafiré : Le climat de la région de Ferké située au Nord de la Côte d'Ivoire est de type tropical sec avec deux saisons : l'une sèche, de novembre à avril et l'autre humide, de mai à arrosages. Les principales hypothèses de travail à tester sont les suivantes:

- L'Analyse fréquentielle des pluies montre une répartition décadaire de la pluviométrie annuelle très différente pour les années sèche, médiane et humide dont les fréquences sont respectivement égales à 8 ans/10, 5ans/10 et 2 ans/10 ;

- $\quad$ Les variations de l'indice de Nicholson et de la moyenne mobile des pluies montrent une atténuation de la pluviométrie sur la période allant de 1999 à 2014.

- Le déficit pluviométrique cumulé sur la période sèche concernée par la pratique de l'irrigation est d'environ $200 \mathrm{~mm}$; celle-ci débute de novembre à mars.

octobre. Le régime pluviométrique est de type unimodal et centré sur les mois d'août-septembre qui cumulent presque la moitié de la hauteur moyenne annuelle des précipitations égale à environ $1200 \mathrm{~mm}$. Le déficit pluviométrique à combler par l'irrigation pour satisfaire les besoins en eau de la canne à sucre approche en moyenne les $700 \mathrm{~mm}$ (Péné et al, 2012c). La saison sèche est marquée par une période très favorable à la maturation de la canne à sucre, celle de l'harmattan qui s'étend de mi-novembre à fin janvier, avec des écarts thermiques journaliers au-delà de $20^{\circ} \mathrm{C}$ et une humidité relative de l'air atteignant parfois $30-35 \%$. 


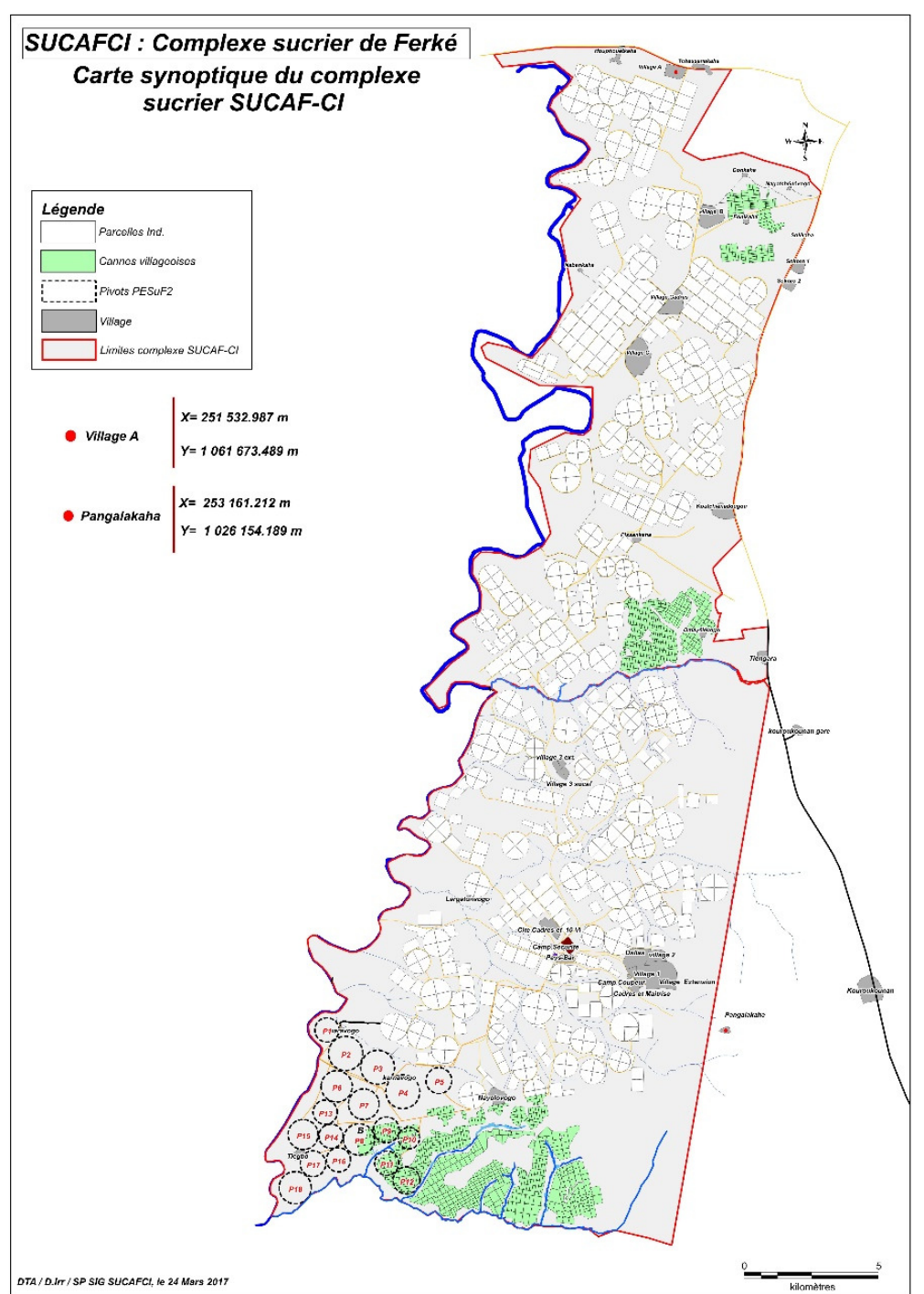

Fig 1. Carte du parcellaire des deux complexes sucriers de Ferké, Nord Côte d'Ivoire. Field map of Ferké sugarcane plantations in Northern Ivory Coast.

Géomorphologie : Le sous-sol des périmètres sucriers de Ferké sont constitué de roches métamorphiques et ignées du Précambrien (faisant partie du complexe de base où dominent les granites et les gneiss granitiques). Du point de vue minéralogique, il y a une dominance de muscovite et de biotite. Les affleurements de granites et de gneiss montrent que le degré de métamorphisme semble avoir été très variable. Ces affleurements s'observent selon une bande parallèle au Bandama, mais aussi par endroits dans d'autres secteurs de la zone. La topographie des 2 périmètres sucriers est ondulée, avec de longues pentes douces dépassant rarement une déclisivité de $5 \%$ et des interfluves généralement surmontés de granites ou de gneiss. L'altitude varie entre 280 et $380 \mathrm{~m}$ au-dessus du niveau de la mer et l'amplitude des séquences topographiques individuelles est inférieure à $70 \mathrm{~m}$. Les plateaux de cuirasses constituent l'élément le plus élevé de la séquence. La zone est drainée par des affluents de rive du Bandama (Lokpoho, Monongo, Waha, Farakwo), selon un mode dendritique (Avenard, 1971; Tate et Lyle, 1975).

Végétation et unités pédologiques rencontrées : La végétation de la région de Ferké est une savane guinéenne (ou sub-soudanaise) boisée, avec des niveaux variables renfermant de petits lambeaux de forêts détachés. Les sols sont à majorité ferralitique, avec une couche arable peu profonde ( 40 à $60 \mathrm{~cm}$ ) limitée par des indurations (carapaces ou cuirasses). Ces sols sont dérivés essentiellement de roches ignées ou métamorphiques du complexe de base qui ont subi des 
périodes d'altération profonde due aux intempéries, suivies par l'érosion et la dissection au cours des temps géologiques passés (Tate et Lyle, 1975).

Les études pédologiques effectuées par la SOGETHA et I'IRAT ont permis de classer les sols en 5 catégories suivant leur aptitude à la culture de canne à sucre. On distingue, en effet $(\mathrm{RCl}, 1971)$ :

- $\quad$ Classe I : bonne valeur agricole ;

- $\quad$ Classe II : valeur agricole bonne à moyenne ;

- $\quad$ Classe III : valeur agricole moyenne ;

- $\quad$ Classe IV : valeur agricole médiocre ;

- $\quad$ Classe V : valeur agricole nulle.

Les facteurs limitants d'ordre physique des sols appartenant aux classes I, II et III sont :

- Sols inondables

- $\quad$ Sols à réserve en eau utile limitée ;

- Sols à profondeur limitée ;

- $\quad$ Sols chimiquement pauvres.

Les sols en place dans les deux périmètres sucriers, de type ferralitique remanié, avec une texture sabloargileuse de couleur ocre, sont marqués par une induration latéritique à moyenne profondeur $(80 \mathrm{~cm})$. C'est la texture dominante ( $40 \%$ des superficies) avec une réserve en eau utile est de l'ordre $90 \mathrm{~mm}$, soit une réserve facilement utilisable de $60 \mathrm{~mm}$ (Péné et Koulibaly, 2011). Les 2 autres textures dominantes sont sableuse et sablo-argilo-limoneuse, avec respectivement 24 et $20 \%$ des superficies. Le sol est pauvre en matière organique (1.5\%) avec un pH fortement acide (6.0) et une faible capacité d'échange cationique (8 méq/100g).

Caractérisation agro-climatique

Analyse fréquentielle des pluies décadaires à Ferké 2: Elle consiste à constituer d'abord une base de données comportant les 36 cumuls de pluies décadaires correspondant à chacune des 17 années considérées (1999 à 2016). Ensuite, à partir de cette base de données, il s'agit de déduire :

- Une répartition annuelle des cumuls de pluies décadaires de telle manière que la valeur de chaque décade est la plus élevée parmi les 17 cumuls correspondant aux 17 années prises en compte (année dite humide) ;

- Une répartition annuelle des cumuls de pluies décadaires de telle manière que la valeur de chaque décade est la plus faible parmi les 17 cumuls correspondant aux 17 années prises en compte (année dite sèche) ;
- Une répartition annuelle des cumuls de pluies décadaires de telle manière que la valeur de chaque décade est la plus contrée parmi les 17 cumuls correspondant aux 17 années prises en compte (année dite médiane) ;

Variabilité interannuelle de la pluviométrie : indice de Nicholson : La variabilité interannuelle de la pluviométrie peut être exprimée par l'indice de Nicholson, une variable centrée-réduite définie comme suit (Nicholson et al, 1988 cités par Paturel et al, 1997):

$\ln =(X i-M) / \sigma$

Avec :

In : indice de Nicholson;

$X i$ : cumul annuel de pluies pour l'année i ;

$M$ : Moyenne annuelle de la pluviométrie correspondant à la période normale ;

$\sigma$ : écart-type relatif à la pluviométrie moyenne $M$ (période normale).

Cet indice permet de mettre en évidence, pour une série pluviométrique donnée, les périodes de pluies excédentaires (humides) ou déficitaires (sèches). Une période est dite normale lorsque les fluctuations de l'indice de part et d'autre de l'axe des abscisses s'annulent. Dans ce cas, la moyenne annuelle est sensiblement égale à la moyenne de la période de référence. La période est dite excédentaire (ou humide) quand la moyenne annuelle est supérieure à la moyenne de la période normale. Elle est dite déficitaire (ou sèche) quand la moyenne annuelle est inférieure à la moyenne de la période normale. La représentation graphique de l'indice sur une période de 10 ans traduit l'évolution de la pluviométrie dans le temps et permet d'en dégager la tendance.

Bilan hydrique climatique : Le bilan hydrique climatique relatif à une période donnée est calculé à partir des valeurs moyennes mensuelles de la pluviométrie et de l'évapotranspiration potentielle (ETP) comme suit :

$\mathrm{BHC}=\mathrm{P}-\mathrm{ETP}$

Avec:

$\mathrm{BHC}$ : bilan hydrique climatique ;

$\mathrm{P}$ : pluviométrie moyenne ;

ETP : évapotranspiration potentielle moyenne.

$B H C \geq 0$ si $P \geq E T P$ : les conditions climatiques sont favorables à la croissance et au développement des cultures;

$\mathrm{BHC}<0$ si $\mathrm{P}<\mathrm{ETP}$ : les conditions climatiques sont défavorables à la croissance et au développement des cultures. L'équation du bilan hydrique climatique de la parcelle qui, par définition, ne tient pas compte des termes étroitement liés au sol tels que le ruissellement, le 
drainage et l'évapotranspiration réelle de la culture est la suivante :

Bilan $=P+I-$ ETM

Avec $P$, la pluviométrie enregistrée, I, l'irrigation apportée et ETM, l'évapotranspiration maximale de la canne à sucre, produit du coefficient cultural kc par l'évaporation $\mathrm{du}$ bac classe A (Evbac). Les valeurs de kc, qui varient selon le stade phénologique de la canne à sucre, sont égales à $0.5,1.0$ et 0.8 respectivement durant 2,8 et 1 mois. La pluie utile est estimée à $80 \%$ de pluviométrie enregistrée lorsque celle-ci est supérieure à $20 \mathrm{~mm}$. L'eau totale utile calculée sur une période donnée est la somme de la pluie utile et des irrigations apportées. Par hypothèse, le ruissellement de l'eau d'irrigation sous culture est négligeable étant donné la faible intensité des apports par rapport à la perméabilité du sol. Enfin dans le calcul du bilan hydrique climatique, les pertes d'eau de pluie ou d'irrigation par percolation profonde sous le front racinaire $(60 \mathrm{~cm})$ ne sont pas prises en compte. Dans le cadre du pilotage de l'irrigation, la réserve en eau utile prise en compte dans la détermination de la dose maximale d'arrosage d'une parcelle donnée a été évaluée comme suit :

$\mathrm{RU}(\mathrm{mm})=\left(\mathrm{Hp}_{\mathrm{cc}}-\mathrm{Hp} \mathrm{p}_{\mathrm{fp}}\right) \times \mathrm{Da} \times \mathrm{Z}$

Avec:

$\mathrm{Hp}_{\mathrm{cc}}$ : humidité pondérale du sol à la capacité au champ $(\mathrm{g} / \mathrm{g})$, déterminée au laboratoire à l'aide d'une presse à plaques ;

\section{RÉSULTATS}

Analyse fréquentielle des pluies décadaires à Ferké 2: Les cumuls pluviométriques correspondant aux années sèche, médiane et humide sont respectivement égaux à 204.3, 1250.8 et $3424.9 \mathrm{~mm}$ (figure 1). Pour ces 3 années fictives (sèche, médiane et humide), les cumuls relatifs à la période critique d'alimentation en eau de la canne à sucre allant de mars à juillet sont respectivement égaux à $67.5,597$ et $1540.3 \mathrm{~mm}$. Le régime pluviométrique à Ferké $2 \mathrm{a}$ une allure unimodale centrée
$\mathrm{Hp}_{\text {fp }}$ : humidité du sol au point de flétrissement permanent $(\mathrm{g} / \mathrm{g})$, déterminée au laboratoire à l'aide d'une presse à plaques ;

Da: densité apparente sèche du sol $(\mathrm{g} / \mathrm{cm} 3) /(\mathrm{g} / \mathrm{cm} 3)$, déterminé au laboratoire après échantillonnage du sol au champ à l'aide d'un cylindre de prélèvement de sol non remanié de volume connu ;

$Z$ : profondeur d'enracinement $(600 \mathrm{~mm})$, déterminée suite à des observations de profils racinaires dans les champs de canne à sucre.

Détermination des périodes humides ou sèches selon Franquin (1969) : La représentation graphique conjointe des variations de la pluviométrie et de l'ETP moyennes mensuelles sur une année selon Franquin (1969) permet de déterminer les périodes humiques d'une part et sèches d'autre part et de présenter ainsi le découpage saisonnier du calendrier agricole. La période humide correspond au cas où la pluviométrie moyenne mensuelle est supérieure à ETP/2. Elle couvre la préparation des semis ou plantations jusqu'à la maturité des produits agricoles. La période sèche, par contre, correspond au cas où la pluviométrie moyenne mensuelle est inférieure à ETP/2. Elle couvre les travaux de récolte, de séchage et de conditionnement des produits agricoles.

en août-septembre avec des valeurs (pour ces deux mois) de 481.8 et $673 \mathrm{~mm}, 190.7$ et $227.3 \mathrm{~mm}, 56.7$ et $71.0 \mathrm{~mm}$, respectivement pour les années humide, médiane et sèche. La période critique (de mars à juillet) se caractérise par une pluviométrie très variable d'une décade à l'autre. Toutefois, l'année humide montre que les mois de mai et juillet présentent un risque de sécheresse relativement important à prendre en compte dans la conduite de l'irrigation à Ferké 2. 


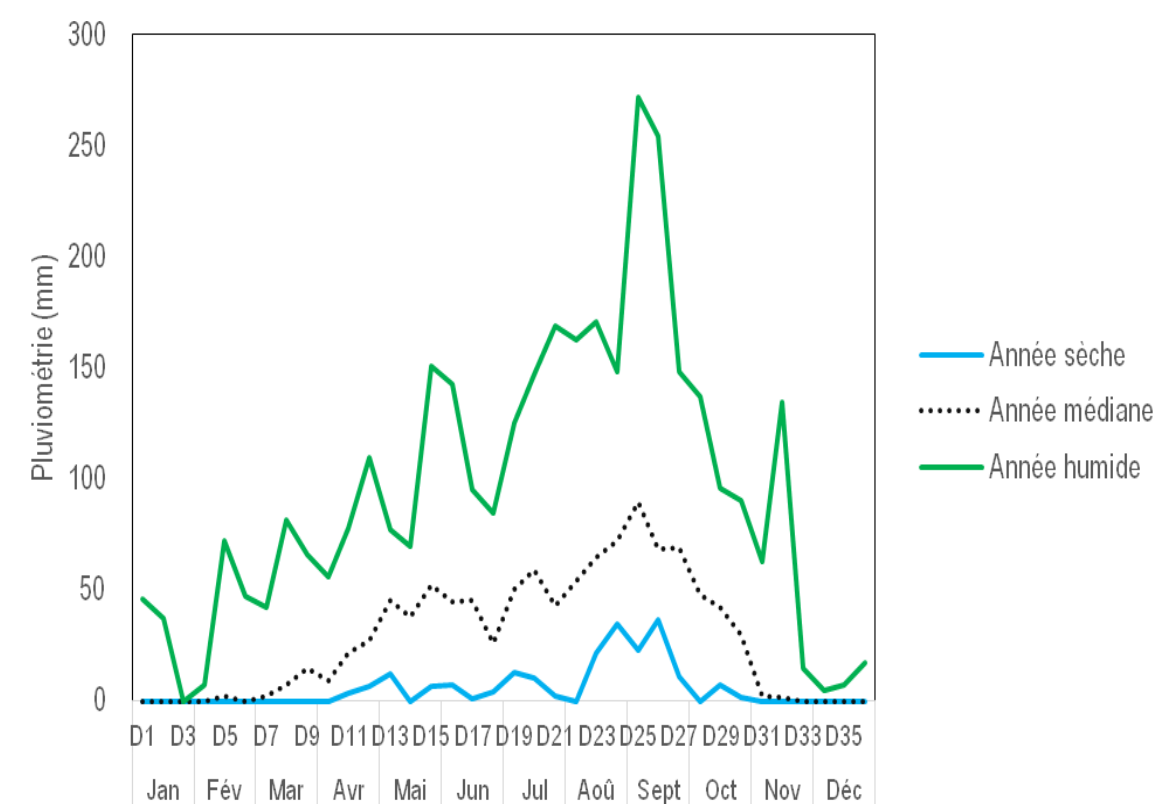

Figure 1: Répartition fréquentielle des pluies décadaires à Ferké 2 pour les années humide (2ans/10), médiane (5ans/10) et sèche (8 ans/10) à partir de la série pluviométrique 1999-2014.

10-day rainfall distribution in Ferké 2 sugar Complex regarding wet (2yrs/10), median (5yrs/10) and dry (8yrs/10) years of 1999-2014 rainfall series.

\section{Moyennes pluviométriques à Ferké 2}

Période décennale 1999-2009 : Le cumul moyen annuel de pluies sur la période décennale 1999-2009 est égal à $1282.5 \mathrm{~mm} \pm \mathrm{mm}$ tandis celui sur la période critique pour l'alimentation hydrique de la canne à sucre est de 638.3 $\mathrm{mm}$. Le nombre de jours de pluies dans l'année de hauteur $\geq 6 \mathrm{~mm}$ est égal à $53 \mathrm{j}$ dont $26 \mathrm{j}$ sur la période critique.

Période quinquennale 2010-2014 : Le cumul moyen annuel de pluies sur la période quinquennale 2010-2014 est égal à $1199.3 \mathrm{~mm} \pm \mathrm{mm}$ tandis celui sur la période critique pour la canne à sucre est de $517.3 \mathrm{~mm}$. Le nombre de jours de pluies dans l'année de hauteur $\geq 6$ mm est égal à $53 \mathrm{j}$ dont $23 \mathrm{j}$ sur la période critique.

II apparaît que cette seconde période de 5 ans montre une baisse de la pluviométrie par rapport à la période 1999-2009 (10 ans), en termes d'épisodes pluvieux mais aussi de cumul annuel, soit $3 \mathrm{j}$ et environ $80 \mathrm{~mm}$ en moins.

Tendance pluviométrique sur la période 1999-2014: La moyenne pluviométrique sur la période 1999-2014, soit une quinzaine d'années, donne $1256.5 \pm 165 \mathrm{~mm}$ et environ 4 jours de pluies de hauteur $\geq 6 \mathrm{~mm}$ dans le mois. La pluviométrie maximale atteinte sur cette période a été obtenue en 2011 avec $1547.7 \mathrm{~mm}$ et la plus faible en 2004 avec $988 \mathrm{~mm}$. Le cumul moyen annuel de l'ETP donne $1433 \mathrm{~mm}$, soit un déficit hydrique climatique d'environ $177 \mathrm{~mm}$ (figures 2 et 3). Le chevauchement de la courbe relative à ETP/2 avec les histogrammes des pluies moyennes mensuelles montre que la saison humide débute en avril et se termine en octobre. La représentation graphique de la pluviométrie moyenne mobile confirme la tendance à la baisse indiquée plus haut (figure 4). Cette tendance est également corroborée par les variations de l'indice de Nicholson (figure 5) 


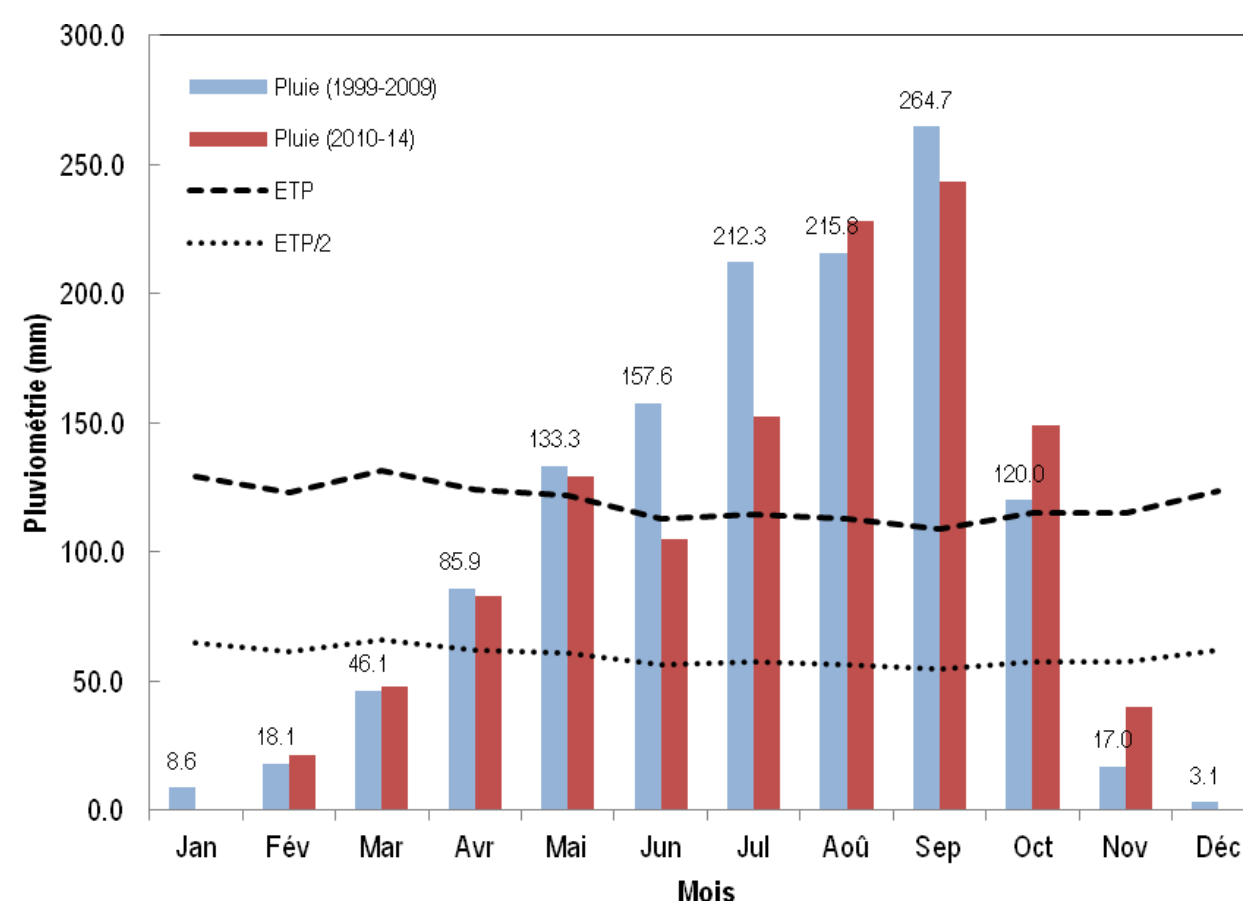

Figure 2. Répartition de la pluviométrie et de l'ETP moyennes mensuelles dans l'année à Ferké 2 sur deux périodes distinctes (1999-2009 et 2010-14).

Average monthly Rainfall and PET distributions over a year in Ferké 2 regarding two periods (1999-2009 and 2010-14).

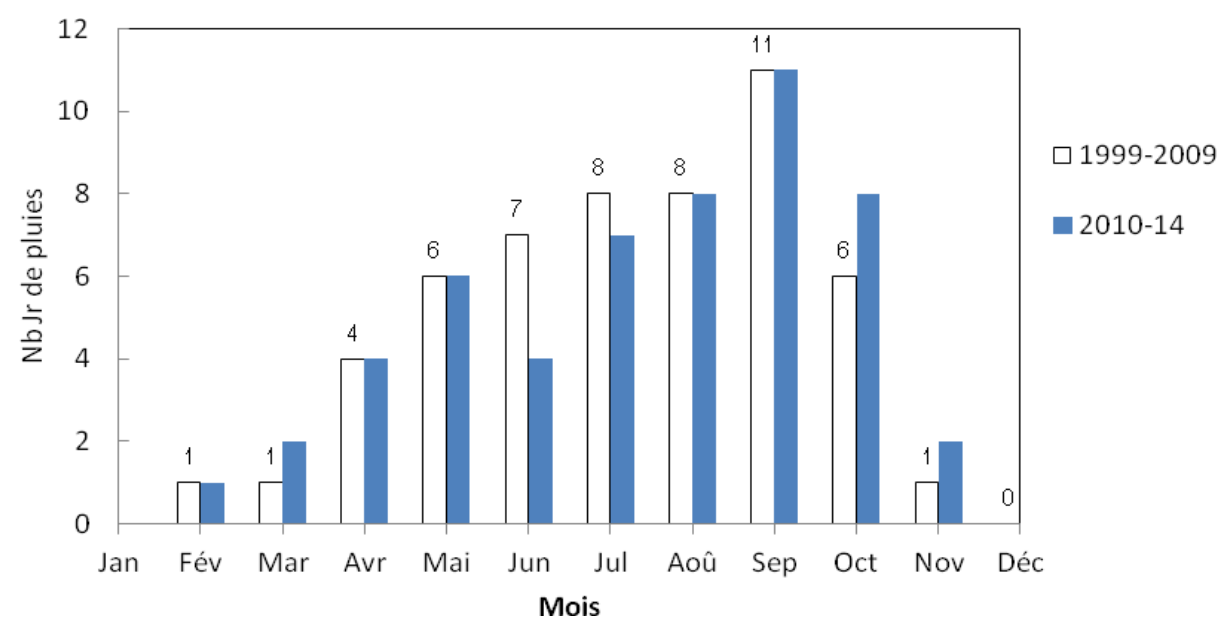

Figure 3. Répartition du nombre moyen mensuels de jours de pluies dans l'année à Ferké 2 sur deux périodes distinctes (1999-2009 et 2010-14).

Average monthly day of rainfall distribution over a year in Ferké 2 regarding two periods (1999-2009 and 2010-14). 


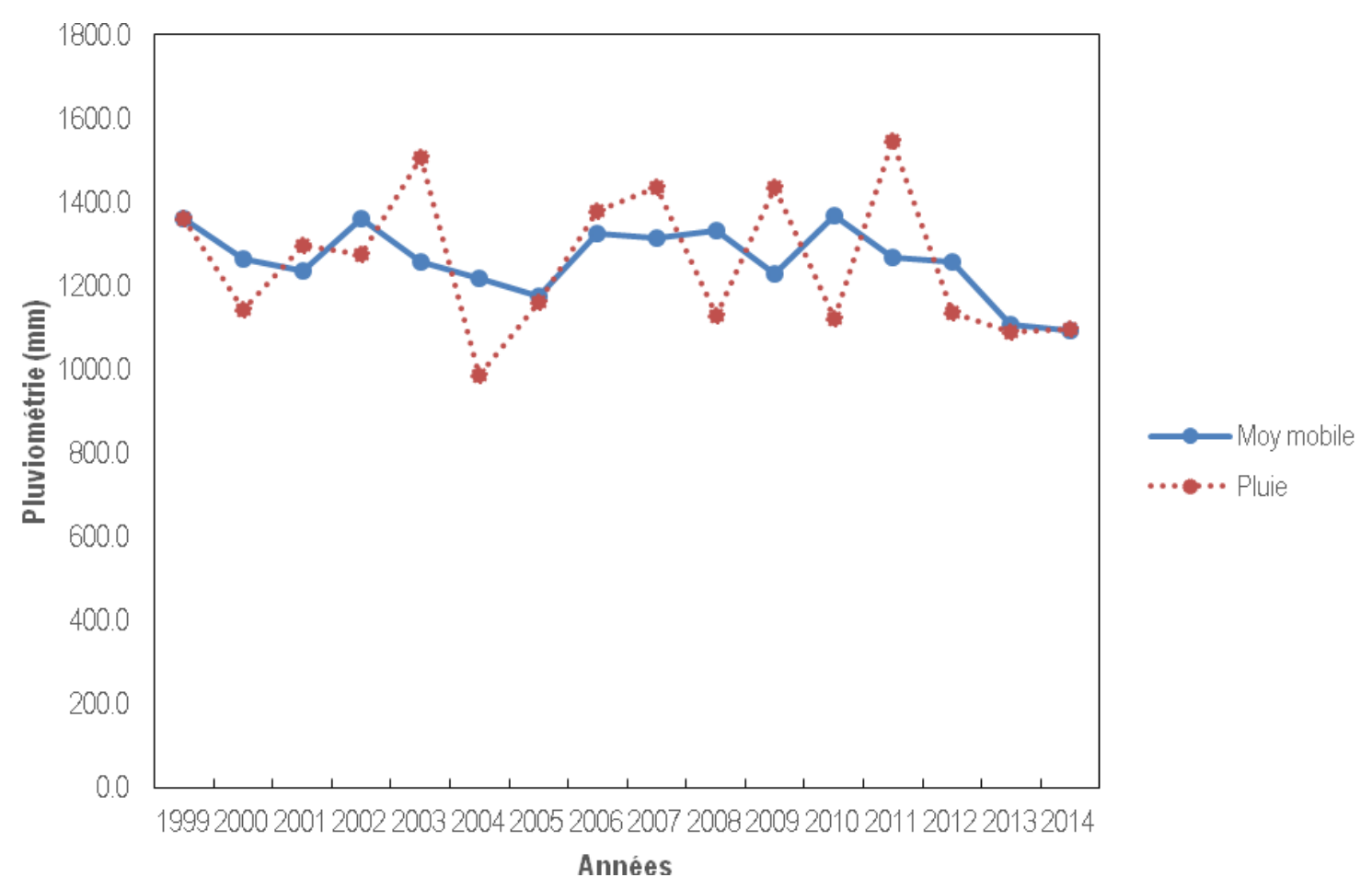

Figure 4. Variations de la moyenne mobile de la pluviométrie annuelle de Ferké 2 sur la période 1999-2014. Running average of annual rainfall variations in Ferké 2 over the 1999-2014 period.

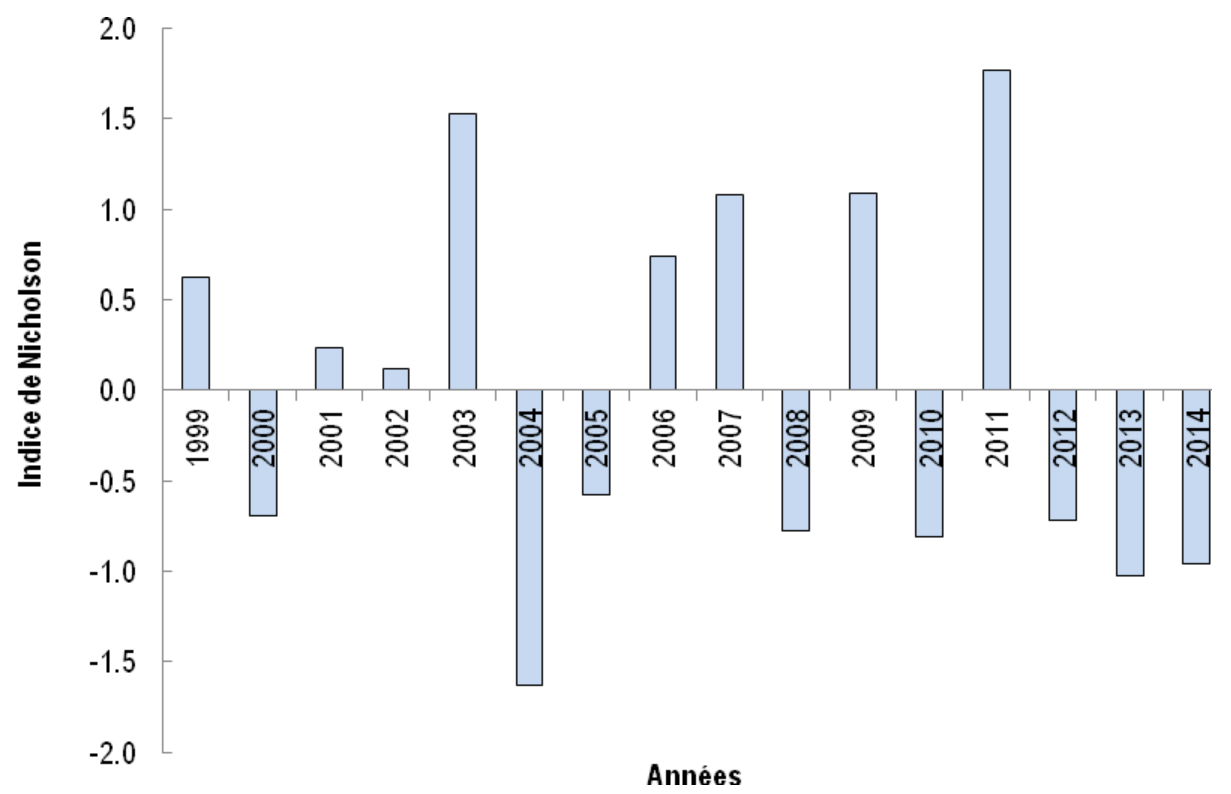

Figure 5. Variations de l'indice de Nicholson sur la série pluviométrique relative à Ferké 2 (1999-2014). Nicholson index variations of Ferké 2 1999-2014 rainfall series. 


\section{DISCUSSION}

Récession pluviométrique en Côte d'Ivoire : Plusieurs études sur les tendances pluviométriques régionales en Côte d'Ivoire ont déjà été réalisées qui concluent à une baisse généralisée des précipitations pour l'ensemble des zones savanicoles et forestières, les zones côtières ayant été touchées dès le début des années 1970 (Servat et al, 1997; Bigot et al, 2005). À partir d'un réseau plus complet comprenant 42 séries pluviométriques sur la période 1950-1996, une analyse en composante principale $(A C P)$ a été réalisée sur les données centrées. Cette étude a montré si la baisse générale des précipitations est sans conteste le fait statistique majeur de la climatologie ivoirienne des trois dernières décennies, elle ne correspond qu'à environ $38 \%$ de la variance totale de la pluviométrie. Au-delà de cette évolution générale, le comportement interannuel de l'espace littoral se distingue de celui des régions intérieures, les anomalies pluviométriques de cette zone étant souvent moins intenses (exemples des épisodes anormalement humides au milieu des années 1950, ou normalement secs dans les années 1980). Cela peut être dû a priori à l'utilisation des valeurs centrées qui donnent plus de poids aux stations littorales enregistrant les plus forts écarts à la moyenne. Mais les analyses factorielles effectuées sur les valeurs centrées-réduites indiquent en fait les mêmes résultats cela prolonge les résultats de Paturel et al (1997) qui avaient déjà observé que la baisse des précipitations au cours des années 1980 (entre 18 et $20 \%$ du total annuel moyen) était surtout représentative des régions nord et côtières des pays en bordure du Golfe de Guinée. La baisse des précipitations est surtout sensible dans le sud-est du pays, même s'il est difficile d'établir une limite précise, étant donné l'anisotropie situationnelle et le manque de postes dans les régions septentrionales. La diminution affecte en fait aussi les régimes typiquement bimodaux du sud que les régimes unimodaux de la partie septentrionale. Alors que le domaine privilégiant théoriquement la couverture arborée sèche $(<1300 \mathrm{~mm} / \mathrm{an})$ s'étend, celui favorable à la forêt tropicale humide (>1300 mm/an) se rétracte, aussi bien dans e sud que dans l'Ouest du pays à l'échelle de plusieurs décennies et en fonction de la modification de seuils économiques, cela peut être la cause fondamentale de changements phytogéographiques majeurs, notamment le glissement vers le sud de la mosaïque forêt-savane pré-forestière, conformément à l'idée ancienne de "savanisation » du domaine semidécidu (Aubreville 1938, Spichiger and Pamard, 1973). Mais cette vision strictement bioclimatique, largement inspirée de la notion de "végétation climacique» évoluant vers des formes altérées, est complètement remise en cause, tant sur le plan historique que sur celui de la prise en compte d'observations botaniques (Fairhead, Leach, 1998). Face au poids des variations climatiques en zone tropicale humide, le rôle des sociétés reste souvent prépondérant et explique une large part des différenciations phytogéographiques régionales. En outre, l'étude des variations pluviométriques mensuelles récentes révèle un certain nombre de particularismes régionaux indiquant qu'il est difficile d'établir des conclusions valables pour l'ensemble du pays et tout au long de l'année. Plusieurs situations apparaissent :

- la baisse des précipitations est faible, sans configuration spatiale claire (janvier, février et mars) ;

- la baisse des précipitations est surtout circonscrite à l'espace littoral (mai, juillet, novembre et décembre, au sud-est (avril ou à l'ouest du pays (septembre) ;

- les précipitations diminuent fortement et pratiquement dans l'ensemble du pays (juin et octobre) ;

- les précipitations augmentent dans le sud et surtout l'ouest du pays (août).

Influence de la variabilité thermique atlantique sur les pluies en Côte d'Ivoire

À partir d'une approche multivariée sur une base saisonnière, Kouadio et al (2002) ont montré qu'un refroidissement des températures de surface de l'océan (TSO) dans le Golfe de Guinée en mai, au début de la grande saison des pluies, précède une hausse des précipitations sur le littoral ivoirien. Afin de mieux tenir compte des caractéristiques régionales de ces éventuelles corrélations, la méthode de la Singular Value Decomposition (SVD) permet de comparer simultanément les champs thermiques de surface de océan Atlantique discrétisés en points de grille de $2^{\circ} \times 2^{\circ}$ (données NOAA-CIRES-CDC) et les données pluviométriques station elles sur la période 1950-1996. Seuls les trois premiers modes de la SVD $(81.5 \%$ de la covariance totale) peuvent être considérés comme statistiquement significatifs au seuil de $95 \%$, d'après le test de Monte Carlo fondé sur 10000 simulations :

- le 1 er mode (12.3\% de la covariance totale) correspond à une baisse généralisée des précipitations en Côte d'Ivoire, parallèlement à un réchauffement anormal des TSO de l'Atlantique sudouest alors que les TSO au large de la Guyane se refroidissent, la relation étant particulièrement significative en hiver nord-hémisphérique. Ce mode couplé explique une part important de la tendance à la baisse des précipitations en Côte d'Ivoire 
observée après 1970. Cependant, le choix de la fenêtre géographique dans l'analyse factorielle influence la configuration de ce premier mode océanique atlantique, l'étude d'une fenêtre plus étendue vers l'ouest et vers le nord modifiant l'aspect et la position du dipôle thermique océanique normalement signalé plus à l'est (Kouadio et al, 2002) ;

- Le second mode indique qu'une part des variations pluviométriques du centre de la Côte d'lvoire, entre $6^{\circ} \mathrm{N}$ et $9^{\circ} \mathrm{N}$, particulièrement en juillet-août, est corrélée positivement aux changements des TSO équatoriales atlantiques, alors que le nord du pays enregistre parallèlement une corrélation négative. Au-delà du caractère très régional de cette relation, la faible part de variance des précipitations expliquée par ce mode $(5.5 \%)$ provient aussi de la baisse générale observée sur le 1er mode, tendance qui absorbe la majeure partie du signal pluviométrique ;

- Le 3ème mode suggère que l'augmentation des précipitations du sud-est de la côte d'Ivoire est liée à la baisse des TSO de l'Atlantique tropicale au nord de l'Équateur, et inversement. Mais la part de la variance expliquée demeure également faible pour les précipitations $(3.5 \%)$, indiquant finalement l'existence d'autres forçages potentiels, océaniques et/ou atmosphériques, des pluies en Côte d'Ivoire.

Ces résultats statistiques confirment cependant l'existence de relations significatives entre les anomalies thermiques de l'océan Atlantique tropical et certaines évolutions interannuelles régionales des précipitations ivoiriennes (Bigot et al, 2002). Ils montrent par exemple que le changement de gradient thermique saisonnier inter hémisphérique enregistré après 1973-1975 dans l'Atlantique (mode 1) pourrait être en partie corrélé à la baisse des précipitations observées depuis 30 ans en Côte d'Ivoire. Les modes 2 et 3 correspondent respectivement aux variations équatoriales atlantiques de type El Nino et au dipôle méridien observé par Servain et al (1998). Par conséquent, lorsque l'océan Atlantique équatorial et sud est anormalement chaud, cela implique une diminution de l'intensité de la circulation de Hadley sud, et une position plus méridionale de la zone de convergence intertropicale (ZCIT). Les ascendances se forment alors au-dessus des eaux les plus chaudes, tout en déterminant les vents de surface qui convergent vers la ZCIT. Cela conduit à une diminution des précipitations au nord ou une augmentation de celles-ci au sud d'environ $10^{\circ} \mathrm{N}$, modifiant la nébulosité et les conditions hydro-climatiques saisonnières en Côte d'Ivoire, et donc le recyclage de l'eau et les bilans écologiques des différents milieux arborés. Cependant, malgré cette mise en évidence de télé-connexions océano-atmosphériques à l'échelle du Bassin Atlantique, il ne faut pas perdre de vue que seulement un tiers de la variance totale des précipitations ivoiriennes est expliqué par trois modes océaniques. Cela peut provenir du fait que la SVD est appliquée à une fenêtre géographique trop petite, ne tenant pas compte d'autres influences océaniques potentielles. Mais pour une autre région d'étude du Golfe de Guinée (Cameroun et Gabon), Camberlin et al, (2001) montrent également qu'il est difficile d'isoler plus de $40 \%$ de la variance commune entre les variations des TSO globales et des précipitations en Afrique. En outre, plusieurs travaux récents indiquent que l'explication liée au balancement anormal de la ZCIT, en relation avec certains modes de $\mathrm{S}$ TSO, n'est pas suffisante pour comprendre l'ensemble de la variation des précipitations en Afrique de l'Ouest, notamment sa position méridionale qui expliquerait les périodes anormalement sèches (le Barbé et al, 2002).

Stratégies d'adaptation pour la culture cannière à Ferké : Durant une année pluviométrique normale (1200 $\mathrm{mm} / \mathrm{an}$ ), les deux retenues hydo-agricoles de Ferké suffisent à peine pour l'irriguer les parcelles de canne à sucre dans les deux périmètres sucriers de Ferké 1 et de Ferké 2. Cela tient au fait que leur capacité nominale de stockage a significativement baissé pour cause d'envasement puisqu'ils n'ont jamais fait l'objet de curage depuis leur création au début des années 1970. On estime leur taux d'envasement à $15-20 \%$, de sorte qu'ils se remplissent durant la saison des pluies à $80-85 \%$ de leur capacité nominale qui est de 79 millions de $\mathrm{m} 3$, pour le barrage du Bandama et de 11 millions de $\mathrm{m} 3$ pour celui du Lokpoho, soit un volume total de 74 millions de $\mathrm{m} 3$ comme ressources en eau disponibles pour l'irrigation et les besoins domestiques et d'usinage de la canne à sucre et en tout début de campagne sucrière. Avec le projet d'extension du périmètre de Ferké 2 sur 1500 ha irrigués à mettre en œuvre de 2017-18 à 2020-21, il va sans dire que la pression de l'irrigation sur la retenue du Bandama sera relativement forte. C'est pourquoi, la sécurisation des ressources en eau à terme nécessitera les mesures ci-après :

- Mettre en œuvre à moyen terme le projet de curage des deux retenues afin de restaurer leurs capacités nominales de stockage ;

- Ré-profiler et assurer le revêtement des berges des deux principaux cours d'eau drainant les périmètres de Ferké 1 et de Ferké 2, évitant ainsi qu'ils demeurent indéfiniment à l'état de fleuves sauvages 
et vulnérables aux effets néfastes des changements climatiques ;

- Rechercher les nappes aquifères existantes au sein des deux périmètres sucriers afin de les exploiter comme autre ressource en eau plus ou moins renouvelable pour la pratique de l'irrigation en appui aux ressources en eau renouvelables de surface ;

- Sélectionner des variétés tolérantes voire résistantes à la sécheresse non seulement pour la culture pluviale ou villageoise de canne à sucre, mais aussi pour la culture irriguée ;

- Promouvoir des systèmes d'irrigation efficients, limitant au strict minimum les fuites d'eau comme les rampes pivotantes ou l'irrigation localisée (goutte à goutte) après avoir naturellement résolu le problème du colmatage organique ou microbien inhérent à ce système (décantation de l'eau brute et préfiltration de celle-ci avant admission dans le système de filtration de chaque installation) ;

- Valoriser sous forme d'irrigation gravitaire par ruissellement les pertes d'eau générées lors du fonctionnement des rampes pivotantes au profit des parcelles pluviales jouxtant ces équipements ;

- Collecter, chaque fois que la topographie du terrain le permet, les eaux pluviales de ruissellement au profit des parcelles pluviales.

Problématique de l'accès à une eau raréfiée : Au cours des dernières décennies, la pluviosité annuelle a baissé de façon sensible. Avant la décennie 1970 (1950-1969), le volume annuel moyen précipité était partout supérieur à $1000 \mathrm{~mm}$, avec des maxima annuels moyens même largement supérieurs (>1400 mm) le long de la dorsale montagneuse guinéenne et à proximité du littoral. Cette situation contraste considérablement avec ce qui est observé ultérieurement (1970-1999) où la plupart des stations enregistrent des niveaux de précipitations plus faibles que par le passé. En effet, initialement confinée au quart nord-est, la zone de précipitations inférieures à $1200 \mathrm{~mm} / \mathrm{an}$ atteint désormais le Centre-Sud à la latitude de Tiassalé et le Centre-Ouest incluant les stations de Bouaflé, de Daloa et de Séguéla. C'est autour de Dabakala et de Bouna que la situation est la plus préoccupante. Dorénavant, les pluies annuelles y restent généralement inférieures à $1000 \mathrm{~mm} / \mathrm{an}$. L'ambiance climatique y est donc de plus en plus proche de celle des climats tropicaux secs. Cette diminution brutale de la pluviosité touche aussi les stations du Sud, notamment

\section{CONCLUSION}

Les cumuls pluviométriques correspondant aux années sèche, médiane et humide sont respectivement égaux à une partie Sud-Ouest à la limite de Soubré et de Gagnoa ainsi que littoral centre à Sassandra. Dans ces stations qui comptaient parmi les plus pluvieuses, les hauteurs d'eau ne dépassent plus les $1400 \mathrm{~mm} / \mathrm{ans}$. Quelques stations échappent à cette situation de baisse généralisée des quantités d'eau précipitée annuellement. II s'agit en particulier de la station de Tabou, la plus arrosée du territoire, où les hauteurs annuelles restent supérieures à $2400 \mathrm{~mm} / \mathrm{an}$. La variation relative calculée entre les années 1950 à 1960 et les années 1970 à 1990 permet de noter que la diminution des précipitations atteint environ $25 \%$ sur l'ensemble du pays, voire un peu plus dans la partie septentrionale où elle peut atteindre $28 \%$. Malgré la survenance de deux années humides récentes 1994 et 1999 qui ont apporté un espoir de rémission de la sécheresse, les différents tests statistiques et la répartition dans le temps des années sèches et humides ont permis de conclure que la sécheresse n'était pas encore terminée en fin 2002 (Gil, Yann, 2002 et 2004). Bien que la Côte d'Ivoire soit située en zone tropicale humide, cette sécheresse caractérisée par un allongement exceptionnel de la saison sèche qui affecte de nombreux pays d'Afrique de l'Ouest s'y fait également ressentir. Les effets de la récession pluviométrique récente sur la ressource en eau se traduisent, pendant les longues périodes de sécheresse, par une baisse notable, de plus en plus fréquente, du débit des cours d'eau et du niveau des nappes phréatiques, allant jusqu'à l'assèchement (Servat et al, 1997, 1998). D'autres auteurs (Savanné et al, 2001), dans une étude sur la variabilité climatique régionale et de son impact sur l'alimentation des aquifères souterraines entreprises en Côte d'Ivoire, parviennent à des résultats similaires. À partir des coefficients de tarissement calculés à l'aide de la méthode dichotomique de classification hiérarchique et par quantification des volumes mobilisés par les aquifères, ils montrent en effet que la baisse des hauteurs d'eau précipitées se ressent sur les niveaux piézométriques comme sur les volumes mobilisés. Certains bassins versants sensibles tels que celui du Cavally, réagissent immédiatement à cette baisse tandis que d'autres moins sensibles tels que le Drou, réagissent également mas avec un certain retard. Cette situation constitue une préoccupation importante pour les populations ivoiriennes en majorité rurale et faiblement desservies par le réseau moderne d'adduction d'eau.

$204.3,1250.8$ et $3424.9 \mathrm{~mm}$. Le régime pluviométrique à Ferké 2 a une allure unimodale centrée en août- 
septembre avec des valeurs (pour ces deux mois) de 481.8 et $673 \mathrm{~mm}, 190.7$ et $227.3 \mathrm{~mm}, 56.7$ et $71.0 \mathrm{~mm}$, respectivement pour les années humide, médiane et sèche. La période critique (de mars à juillet) se caractérise par une pluviométrie très variable d'une décade à l'autre. Toutefois, l'année humide montre que les mois de mai et juillet présentent un risque de sécheresse relativement important à prendre en compte dans la conduite de l'irrigation à Ferké 2. La période quinquennale 2010-14 montre une baisse de la pluviométrie par rapport à la période décennale 1999-

\section{RÉFÉRENCES BIBLIOGRAPHIQUES CITÉES}

Aubreville A, 1938. La forêt coloniale: les forêts de l'Afrique occidentale française. Annales d'Académie des Sciences Coloniales IX. Paris : Sociétés d'éditions géographiques, maritimes et coloniales, $245 \mathrm{p}$.

Avenard JM, 1971. Aspect de la géomorphologie. In : Le milieu naturel de la Côte d'Ivoire. Mémoires ORSTOM, $n^{\circ} 50$. Paris, ORSTOM: 11-72.

Bigot S, Brout YT, Bonnardot V, Servat E, 2002. Interannual stability of rainfall patterns in the Ivory Coast over the period 1950-1996. Publications of the international Association of Hydrological Sciences 274: 507-517.

Bigot S, TY Brou, Oszwald J, Diedhiou A, 2005. Facteurs de la variabilité pluviométrique en Côte d'Ivoire et relations avec certaines modifications environnementales. Sécheresse 16 (1) : 5-13.

Brou YT, Akindès F, Bigot $S, 2005$. La variabilité climatique en Côte d'Ivoire: entre perceptions sociales et réponses agricoles. Cahiers Agricultures 14 (6) : 533-540.

Camberlin P, Janicot S, Paccard I, 2001. Seasonality and atmospheric dynamic of the teleconnection between African rainfall and tropical surface temperature: Atlantic vs ENSO. International J of Climatology 21: 973-1005.

Fairhead J, Leach M, 1998. Reframing deforestation, global analysis and local realities: studies in West Africa. London: Routledge, $238 \mathrm{p}$.

Franquin $P, 1969$. Analyse agroclimatique en régions tropicales. Saison pluvieuse et saison humide, applications. Cahiers ORSTOM, Série Biologie $n^{\circ} 9: 5-95$.

Gil M, Yann L, 2004. Sahel: une sécheresse persistante et un environnement profondément modifié. La Météorologie 44: 2-3.
2009, en termes d'épisodes pluvieux mais aussi de cumul annuel, soit $3 \mathrm{j}$ et environ $80 \mathrm{~mm}$ en moins. La moyenne pluviométrique sur la période 1999-2014, soit une quinzaine d'années, donne $1256.5 \pm 165 \mathrm{~mm}$ et environ 4 jours de pluies de hauteur $\geq 6 \mathrm{~mm}$ dans le mois. Le cumul moyen annuel de l'ETP donne $1433 \mathrm{~mm}$, soit un déficit hydrique climatique d'environ $177 \mathrm{~mm}$, avec une saison humide qui débute en avril et se termine en octobre. Enfin, les variations de la moyenne mobile de la pluviométrie annuelle et de l'indice de Nicholson montrent une tendance à la baisse de la pluviométrie à Ferké 2 .

Gil M, Yann L, 2002. Analysis of a sahelian annual rainfall index from 1896 to 2000; the drought continues. Hydrology Sciences 47: 563-672.

Kouadio Y, Ochou DA, Servain J, 2002. Atlantic influence on the rainfall variability in Côte d'Ivoire. Geophysic Research Lett 30: 8005-8012.

Kouamé KD, Péné CB, Zouzou M, 2010. Criblage de variétés commerciales de canne à sucre prometteuses dans le périmètre sucrier de Ferké 2 au Nord de la Côte d'Ivoire : Optimisation de la durée de sélection Sci \& nature 7(1) : 97-106.

Le Barbé L, Lebel T, Tapsoba D, 2002. Rainfall variability in West Africa during the year 1950-1990. Journal of Climatology 2: 187-202.

Paturel JE, Servat E, Kouamé B, Lubès H, Ouedraogo M, Masson JM, 1997. Climatic variability in humid Africa along Gulf Guinea, part II: an integrated regional approach. Journal of Hydrology 191: 1636.

Péné CB, Assa AD, 2003. Variations interannuelles de la pluviométrie et de l'alimentation hydrique de la canne à sucre en Côte d'lvoire. Sécheresse 14(1) : 43-52.

Péné CB et Déa BG, 2000. Interaction eau d'irrigationvariétés de canne à sucre en conditions de rationnement hydrique au nord de la Côte d'Ivoire. Revue Agricole et Sucrière de Maurice 79 (2): 1-21.

Péné CB, ASSA DA et Déa BG, 2001. Interactions eau d'irrigation-variétés de canne à sucre en conditions de rationnement hydrique. Cahiers Agricultures 10(4) : 243-253.

Péné CB, Chopart JL, Assa A, 1997. Gestion de l'irrigation à la parcelle en culture de canne à sucre (Saccharum officinarum $\mathrm{L}$ ) sous climat tropical humide à travers le cas des régions nord et centre de la Côte d'ivoire. Sécheresse 8 (2) : 87-98. 
Péné CB, N'Diaye S Konan NC, 2013. Sprinkler irrigation and soil tillage practices in sugarcane plantations as influenced by soil texture and water storage in northern Ivory Coast. J. of life Sci. 7(3) : 293-301.

Péné $\mathrm{CB}$, Kouamé KD, Dove $\mathrm{H}$, 2016. Incidence des infestations du foreur de tiges Eldana saccharina W. en culture irriguée de canne à sucre selon la variété et la période de récolte en Côte d'Ivoire. $\mathrm{J}$ of Applied Biosciences 102: 9687-9698.

Péné CB, Boua BM, Viremouneix T, 2012a. R579, une variété de canne à sucre très prometteuse en développement sous irrigation et en pluvial à Ferké au Nord de la Côte d'Ivoire. In Actes Congrès Sucrier 2012 AFCAS/ARTAS, lle de la Réunion, 10-14 sept 2012. Disponible sur www.afcas.fr

Péné CB, Boua BM, Viremouneix T. Criblage de variétés de canne à sucre sous irrigation en début de campagne sucrière à Ferké 1 en Côte d'Ivoire sur sol ferralitique selon un nouveau schéma de sélection. In: Actes Congrès Sucrier 2012 AFCAS/ARTAS, lle de la Réunion, 10-14 sept 2012. Disponible sur www.afcas.fr

Péné CB, Ouattara HM, Koulibaly GS, 2012c. Late sugarcane performance as affected by soil water regime at the yield formation stage on commercial farms in northern Ivory Coast. $\mathrm{J}$ of life Sci. Vol 6 (6): 644-651.

Péné $C B$, Kéhé $M, 2005$. Performances de trois variétés de canne à sucre soumises au rationnement hydrique en prématuration au Nord de la Côte d'Ivoire. Agronomie Africaine 17 (1): 7-18.

Péné CB, Tuo K, 2007. Early and late-season screening of sugarcane varieties in northern Ivory Coast. Poster presentation (AGP 34). In: $26^{\text {th }}$ ISSCT World Congress on Sugarcane Proceedings, Durban, July 29 -August 03, 2007. Available on www.luss.org

Savanné I, Coulibaly KM, Gioan P, 2001. Variabilité climatique et ressources en eaux souterraines dans la région semi-montangeuse de man. Sécheresse 12: 231-237.

Servat $E$, Parturel JE, Lubès-Niel $H$, Kouamé $B$, Masson JM, 1997. Variabilité des régimes pluviométriques en Afrique de l'Ouest et du Centre non sahélienne. Comptes Rendus de l'Académie des Sciences 324 : 836-838.

Servat E, Paturel JE, Lubès H, Kouamé B, Ouédraogo M, Masson JM, 1998. Variabilité climatique en Afrique humide le long du Golfe de Guinée. 1ère partie : analyse détaillée du phénomène en Côte d'Ivoire. Journal of Hydrology 191 :1-15.

Servain L, Wainer I, Dessier A, Mc Creary JP, 1998. Modes of climatic variability in the tropical Atlantic. In: Water resources variability in Africa during the XX th century. Publications of the international Association of Hydrological Sciences 252: 45-54.

Spichiger R, Pamard C, 1973. Recherches sur le contact forêt-savane en Côte d'Ivoire : étude du recru forestier sur des parcelles cultivées en lisière d'un ilot forestier dans le sud du pays Baoulé. Candollea 28: 21-37.

Tate et Lyle, 1975. Étude pédologique de la région comprise entre les rivières Waha et Farakwo en Côte d'Ivoire. Hunting Technical Services Limited. $99 \mathrm{p}$.

Zadi M, Turquin L, Péné CB, 2017. Évaluations agronomiques et technologiques des clones de canne à sucre (saccharum officinarum L) présélectionnés au stade ligne à Ferké, Nord Côte d'lvoire. J of Applied Biosci. 115 : 1150211512. 